\title{
Dermatophytosis of Groin and Perianal Area
}

National Cancer Institute

\section{Source}

National Cancer Institute. Dermatophytosis of Groin and Perianal Area. NCI Thesaurus.

Code C34535.

Dermatophytosis involving the stratum corneum of the skin of the groin and perianal area. 